\title{
Tsafon
}

Revue d'études juives du Nord

$81 \mid 2021$

Des synagogues à travers les âges Lieux de prières, lieux d'études et autres fonctions

\section{Les synagogues des Juifs du pape dans le Comtat Venaissin et en Avignon}

\section{Carol lancu}

\section{(2) OpenEdition}

\section{Journals}

Édition électronique

URL : https://journals.openedition.org/tsafon/3728

DOI : $10.4000 /$ tsafon. 3728

ISSN : 2609-6420

Éditeur

Association Jean-Marie Delmaire

Édition imprimée

Date de publication : 1 juillet 2021

Pagination : 49-72

ISSN : $1149-6630$

Référence électronique

Carol lancu, «Les synagogues des Juifs du pape dans le Comtat Venaissin et en Avignon », Tsafon [En ligne], 81 | 2021, mis en ligne le 01 juillet 2021, consulté le 15 septembre 2021. URL : http:// journals.openedition.org/tsafon/3728; DOI : https://doi.org/10.4000/tsafon.3728 


\title{
Les synagogues des Juifs du pape dans le Comtat Venaissin et en Avignon
}

\author{
Carol Iancu*
}

\section{Introduction}

Ancien territoire du sud-est de la France, correspondant à une partie de l'actuel département du Vaucluse, limité par le mont Ventoux, le Rhône et la Durance, le Comtat Venaissin passa sous la domination du SaintSiège en 1274. Philippe le Hardi n'a fait qu'entériner la décision qui avait été déjà prise par Raymond VII, comte de Toulouse, de céder ses titres au pape, lors du traité de Paris, en 1229. La cité-État d'Avignon fut achetée à Jeanne de Provence en 1348, par le pape Clément VI, pour la somme de 80.000 florins. Le pouvoir pontifical perdura dans cette aire géographique jusqu'à son rattachement à la France, en 1791. Les Juifs y furent maintenus et protégés par les souverains pontifes, alors qu'ils furent expulsés définitivement du royaume de France en 1394. De cette longue et singulière histoire il subsiste comme témoins, hormis une riche documentation archivistique, des noms de rues spécifiquement juifs, des cimetières et trois superbes synagogues à Carpentras, à Cavaillon et en Avignon, objets de cette étude. Pour mieux comprendre le destin de ces trois magnifiques lieux de prière et le passé de ceux qui les ont fréquentés, nous nous proposons, dans une première partie, d'appréhender la condition qui leur fut imposée.

\section{La gouvernance pontificale et le statut des Juifs}

Le pape gouverna les deux États d'Avignon et du Comtat par un légat, un cardinal italien qui réside à Rome à partir du XVII ${ }^{\mathrm{e}}$ siècle et qui

\footnotetext{
* Professeur émérite à l'Université Paul Valéry - Montpellier 3, membre d'honneur de l'Académie roumaine.
} 
délègue ses pouvoirs sur place à un vice-légat. Le légat est remplacé en 1693 par une Congrégation romaine, dénommée la Congrégation d'Avignon (son préfet étant le secrétaire d'État en personne). Le vice-légat qui habite Avignon doit faire exécuter les instructions arrivant de Rome, du légat, puis de la Congrégation d'Avignon, et il est secondé par deux autres fonctionnaires qui sont sous ses ordres : un viguier en Avignon et un recteur à Carpentras qui administre tout le Comtat. Les Juifs dépendent de ces personnages, mais sont également soumis au pouvoir des évêques et de l'inquisiteur général. Quelle fut la politique des autorités pontificales à l'égard des Juifs dont la présence est attestée à partir de 1178, mais dont le peuplement remonterait au haut Moyen Âge, comme dans la Provence voisine?

Dans leurs possessions transalpines, jusqu'à la Révolution française et à leur rattachement à la France, la politique des papes fut constituée par un savant dosage d'intolérance - une sévère ségrégation - mais aussi d'une certaine tolérance. Cette ambiguïté qui se retrouve déjà tout au long du Moyen Âge était la conséquence de la position doctrinale forgée par les pères et les princes de l'Église sur le «peuple témoin». Si les Juifs devaient perdurer comme témoins de la révélation chrétienne, en revanche le statut qu'on allait leur imposer ne pouvait être qu'humiliant et dégradant, car ils avaient refusé de suivre la «nouvelle alliance» et le fondateur du christianisme issu de son sein : Jésus de Nazareth. Nous sommes en présence d'une véritable doctrine bipolaire de l'Église : d'une part, la proclamation de la nécessité de la survie du « reste d'Israël » appelé à embrasser le christianisme aux derniers jours, ce qui implique la nécessité de garantir sa sécurité ; d'autre part, l'impatience devant le refus des Juifs de se convertir, d'où la condition humiliante qu'on leur assigne. L'attitude de l'Église se raidit dès le $\mathrm{XV}^{\mathrm{e}}$ siècle et surtout au $\mathrm{XVI}^{\mathrm{e}}$ siècle, marquée par la "Contre-Réforme », terme impropre pour désigner la Réforme catholique, en réaction à la Réforme protestante.

L'intolérance se manifesta par la quadruple discrimination traditionnelle: dans l'habitat, le vêtement, le serment et l'activité économique. La tolérance était concédée dans l'autogouvernement, dans l'autonomie interne des communautés juives qui devait cependant être agréée par les pouvoirs locaux. En somme, une sécurité relative, un asile chèrement payé, marqué de brimades, d'avanies, d'un rigoureux ostracisme et d'essais d'expulsion et de conversion des Juifs.

La ségrégation dans le domaine topographique consiste en la limitation drastique des localités d'habitation et l'enfermement des Juifs 
dans des quartiers isolés de ces localités, dans la ghettoïsation. Présents dans une vingtaine de localités (Aubignan, Avignon, Beaumes, Bédarrides, Bollènes, Caderousse, Carpentras, Cavaillon, Châteauneufdu-Pape, Châteauneuf-de-Gadagne, Entraigues, L'Isle-sur-la-Sorgue, Le Thor, Malaucène, Mazan, Monteux, Mormoiron, Mornas, Pernes, SainteCécile, Vaison, et Valréas), les Juifs furent obligés à partir du XVII ${ }^{\mathrm{e}}$ siècle de résider dans des ghettos (appelés «carrières », du mot provençal carriero, désignant la rue) de quatre villes seulement: Carpentras, Cavaillon, L'Isle et Avignon.

Le deuxième aspect de l'intolérance réside dans la discrimination vestimentaire : la roue ou la rouelle (rota, rotella), imposée par le Concile de Latran de 1215, fut remplacée pour les femmes par un voile particulier (appelé oralia) et pour les hommes par le chapeau jaune, d'après une bulle de 1524, du pape Clément VII. Le chapeau jaune (lou capéou) pour les hommes et la cocarde de la même couleur pour les femmes (appelée aussi lou pecihoun ou patassoun, signifiant un morceau d'étoffe) furent rigoureusement maintenus jusqu'à la Révolution française, malgré de nombreuses récriminations. Durant leurs voyages en France, les Juifs du pape ont pu cependant se coiffer à leur guise.

Le more judaico, appelé le herem de coulbo, est le troisième aspect de l'intolérance officielle : il s'agit d'un serment particulier infamant par la forme et le contenu, auquel les Juifs étaient astreints lorsqu'ils comparaissaient dans des procès. La prestation du serment n'avait pas lieu au tribunal, mais dans une synagogue (ou une salle attenante) et se déroulait dans des circonstances extravagantes : des tentures noires étaient accrochées aux murs, la personne étant revêtue aussi d'un manteau et d'un chapeau noirs et, tandis que des cierges noirs précédemment allumés s'éteignaient progressivement, des chants des morts s'élevaient dans l'assistance. Le rabbin sortait la Tora, faisait poser la main de l'intéressé sur le rouleau de la Loi en lui demandant de répéter des formules injurieuses. À l'instar des tentatives d'abrogation du chapeau jaune, le combat pour l'abrogation de ce serment fut voué à l'échec jusqu'à la fin du régime pontifical.

Le quatrième et dernier aspect de l'intolérance est représenté par des restrictions dans le domaine économique (interdiction de posséder des terres et des immeubles en dehors de la résidence principale dans les carrières, interdiction également de faire le commerce de marchandises neuves ainsi que de denrées alimentaires et d'animaux) qui poussèrent les Juifs vers la friperie, la brocante et le prêt d'argent, occupations principales 
dans les carrières dès le $\mathrm{XVII}^{\mathrm{e}}$ siècle, avec le métier de tailleur, largement pratiqué.

La tolérance s'exerça dans la totale liberté d'une organisation interne (comme un « self-government»), dans l'établissement des institutions communautaires, connues grâce à leurs statuts rédigés en hébreu et appelés escamot. Les quatre communautés ou arba kehilot étaient de véritables républiques censitaires, leur administration était confiée en général à trois baylons, entourés par douze conseillers, choisis dans la catégorie la plus aisée de la population, les pauvres n'étant pas représentés dans les instances communautaires. Il y eut une amélioration de la condition économique des Juifs entre le XVII ${ }^{\mathrm{e}}$ et le $\mathrm{XVIII}^{\mathrm{e}}$ siècles, mais tous les membres des carrières n'ont pas bénéficié, dans une égale mesure, de cette évolution et nombreux furent ceux qui gardèrent le statut d'assistés, tandis qu'une minorité se détachait du reste du groupe, grâce aux affaires menées en dehors des frontières pontificales. En 1782, dans la communauté de Carpentras, le tiers des membres étaient pauvres et inscrits pour la distribution des aides, en face de quelques familles opulentes.

Du point de vue démographique, nous sommes en présence de petites communautés, l'ensemble des Juifs n'a probablement jamais dépassé le chiffre total de 2.500 personnes dont la moitié à Carpentras, capitale du Comtat. En 1787, suite à un mouvement migratoire vers les régions voisines où ils furent tolérés, malgré l'interdiction de séjour officielle, ils étaient seulement environ 400 en Avignon, 900 à Carpentras, 350 à L'Isle-sur-la-Sorgue, et 200 à Cavaillon, pour respectivement $24.000,11.000,5.000$ et 6.000 catholiques.

\section{La carrière et la place de la synagogue}

À la différence de leur situation matérielle générale qui a connu une amélioration progressive, tel ne fut pas le cas de leur condition d'habitation pour les Juifs des arba kehilot. Bien au contraire, il faut constater une évolution inverse : tandis qu'au départ et jusqu'au XVIII ${ }^{\mathrm{e}}$ siècle, les carrières n'étaient pas complètement isolées, notamment pour ce qui est de leur fermeture nocturne et du maintien des passages privés entre les maisons juives et chrétiennes, un siècle plus tard, en raison du poids des édits restrictifs et récurrents, elles sont devenues un monde clos. Les portes du ghetto étaient verrouillées dès la tombée de la nuit et elles étaient gardées, à la fois, à l'intérieur par un gardien juif et à l'extérieur par un gardien chrétien, tous deux rémunérés par la communauté juive. 
Dans chacune des quatre villes catholiques abritant des collectivités juives, la carrière se trouvait en plein centre. Les maisons juives la délimitant avaient toujours leurs fenêtres tournées vers l'intérieur, toute communication avec le monde chrétien extérieur étant prohibée, et tous les passages privés avaient été cloisonnés. La superficie de ces quartiers juifs qui fut toujours trop restreinte par rapport à la population, qui y habitait et s'accroissait régulièrement, n'a pas connu d'agrandissement avant leur disparition définitive. La solution trouvée fut la construction de maisonnettes sur les toits - les immeubles étaient déjà assez hauts (cinq étages en moyenne dans la capitale du Comtat - sans pour autant garantir la sécurité de ces édifices surélevés (il y eut plusieurs effondrements retentissants causant des pertes en vies humaines, notamment en Avignon en 1614 et 1767, et à L'Isle-sur-la-Sorgue en 1754 et 1776. Les difficultés d'accès aux logements supérieurs, où habitaient les plus démunis, poussaient les locataires à utiliser des paniers suspendus à des cordes pour faire monter les marchandises, ce qui augmentait l'insécurité. Dans cet espace fermé, où la promiscuité et l'entassement sévissaient, la rue se transformait en réceptacle des déchets jetés des nombreux étages. Afin d'assurer un nettoyage général régulier, les baylons eurent recours aux services d'un chrétien (paysan ou jardinier) chargé d'appailler l'ensemble des rues, ruelles et impasses : les immondices étaient recouvertes de paille et l'engrais ainsi récupéré était transporté hors la juiverie. Ce fumier semble avoir été très recherché puisqu'il y eut des chrétiens qui se chargèrent de l'entretien de la carrière sans être rémunérés et, parfois même, ce sont eux qui payèrent le droit d'appaillage, comme ce fut le cas à Cavaillon en 1699 et à L'Isle-sur-la-Sorgue en 1775.

L'édifice le plus important de la carrière, représentant le centre et le cœur même de la communauté, a toujours été la synagogue, appelée aussi école (escole).

\section{La synagogue de Carpentras}

\section{Données historiques}

Dans la capitale du Comtat, les Juifs avaient été relégués dans une seule rue dès la fin du $\mathrm{XV}^{\mathrm{e}}$ siècle, auparavant il y eut un premier quartier juif près des remparts du sud-est de la ville dont l'actuelle rue Vieille Juiverie conserve le souvenir. En effet, les Juifs aux XIII ${ }^{\mathrm{e}}$ et $\mathrm{XIV}^{\mathrm{e}}$ siècles, ont trouvé refuge et protection dans le Comtat devenu pontifical en 1274 et, exception faite des pontificats du pape Jean XXII (qui les expulsa en 
1321) et Benoît XII (1334-1342), ont pu y vivre paisiblement. Si l'on ignore le lieu de la première synagogue, en revanche, c'est à partir de leur réinstallation en 1343 qu'il convient de dater l'emplacement de leur quartier en plein centre-ville, la synagogue étant construite deux décennies plus tard, en 1367, avec l'autorisation de l'évêque Jean Roger de Beaufort qui leur accorda aussi un grand terrain pour un nouveau cimetière à l'écart de la ville. En 1461, le Conseil de Ville réduisit de quatre à deux le nombre de rues où ils pouvaient habiter et à partir de 1486 leur quartier fut réduit à une seule rue, l'ancienne rue de la Muse ou rue Pie, d'environ 88 mètres de long, où ils furent cantonnés jusqu'à la Révolution française. Un nombre presque égal de maisons (en tout 168) se trouvait de part et d'autre de cette rue, le quartier juif ayant la forme d'un quadrilatère irrégulier couvrant une superficie de moins de mille mètres carrés. Grâce à deux bénédictins de la Congrégation de Saint-Maur, Martène et Durand, auteurs d'un Voyage littéraire, nous savons comment la synagogue se présentait en 1710 :

... Il y a à Carpentras environ sept cents Juifs, qui sont tous riches [sic !]. Le quartier qu'ils habitent est très vilain, et sent très mauvais. Nous aurions souhaité assister à une de leurs assemblées, mais le peu de temps que nous avions après notre travail ne nous le permit pas. Nous ne voulûmes pas pourtant nous en aller sans voir leur synagogue. Elle est petite et a deux étages. Les hommes sont dans l'étage d'en haut, et les femmes dans celui d'en bas. Ils conservent dans le fonds du premier la Loy dans un tabernacle, avec un rideau qui le cache. Il y a devant une lampe, qui brûle toujours, et un balustre d'où le rabbin lit la Loy à l'assemblée; et tout autour de la synagogue il y a bien deux cens lampes, qui brûlent dans le temps des assemblées. Devant le balustre il y a une petite grille, par laquelle le rabbin montre la Loy aux femmes, qui font alors de grands élancements. Dans leurs assemblées ils chantent des Psaumes en hébreu, dont le chant est charmant (envoûtant). Nous en fîmes chanter un à des petits Juifs, qui nous enlevaient. L'endroit où ils font la circoncision des enfants est hors de la synagogue. ${ }^{1}$

C'est par l'agrandissement du lieu de culte dont les fondations remontent à l'époque médiévale, que l'architecte Antoine d'Allemand, auteur également de l'aqueduc et de l'hôtel-Dieu de Carpentras, édifia de 1741 à 1743, une nouvelle synagogue. Quelque temps après, cet édifice fut abaissé selon les ordres de l'évêque Malachie d'Inguimbert dont l'attitude à l'égard des Juifs fut toujours empreinte d'une grande méfiance

\footnotetext{
${ }^{1}$ Voyage littéraire de deux religieux bénédictins de la Congrégation de Saint-Maur, Paris, 1717, $1^{\text {ère }}$ partie, p. 290.
} 
et animosité, et la construction, telle qu'elle se présente aujourd'hui (après avoir été remise en état en 1929), fut achevée en 1784. Un siècle plus tard, Isidore Loeb dressait le constat suivant dans la Revue des Études juives :

Il est impossible de pénétrer dans la synagogue actuelle de Carpentras sans éprouver une profonde émotion. Elle n'a point de beauté physique [il s'agit de son aspect extérieur], les papes et le clergé y avaient mis bon ordre, elle est faite de pièces et de morceaux, par des additions et restaurations successives, exécutées chaque fois qu'il était possible et qu'on le permettait, mais c'est un édifice vivant, le monument a une âme, chaque pierre raconte les souffrances et les tribulations des pauvres gens qui l'ont élevé, c'est une page d'histoire éloquente et douloureuse. Elle se distingue par de nombreux traits qu'on ne rencontrerait pas facilement ailleurs que dans le Comtat, et peut être, en Italie. On y trouve d'abord un vaste sous-sol, pauvrement éclairé et qui servait autrefois de synagogue pour les femmes. On les reléguait dans cet endroit obscur et froid, parce que la synagogue des hommes, placée au rez-de-chaussée, était trop petite pour les contenir. Elles ne pouvaient suivre l'office fait aux hommes, on leur donnait un officiant spécial, appelé le rabbin des Juives, et qui leur disait les prières, probablement en provençal. Un petit trou, fermé par un grillage, était placé dans le plafond, il s'ouvrait dans le sol de la synagogue des hommes, devant l'arche qui renferme les rouleaux de la Loi ; il était destiné à permettre aux femmes de voir le rouleau de la Loi lorsqu'on le retirait de l'arche pour faire la lecture publique du Pentateuque. Aujourd'hui, cette synagogue inférieure est abandonnée, les femmes assistent aux offices dans les galeries placées à deux ou trois mètres au-dessus du sol de la synagogue des hommes et dans les chambres ou tribunes placées aux angles de la synagogue...2

\section{Description de la synagogue}

La salle du culte, la plus grande de l'immeuble, mesurant $8,5 \mathrm{~m}$ sur $15 \mathrm{~m}$, se trouve au premier étage et comporte un deuxième niveau, une tribune soutenue par des colonnes où l'on accède par un double escalier. C'est là que se situe la tebah (pupitre ou tribune de l'officiant) sur laquelle est placée la Torah, lors de sa lecture publique (les lundis, jeudis, samedis et les autres jours de fête). Devant la tebah, surmontée par un baldaquin, se dressent trois chandeliers à sept branches fixées à un beau grillage de fer forgé qui délimite et sépare la tribune de côté de la salle de prière. Quelques rangées de bancs se trouvent de part et d'autre de l'allée centrale menant de la porte d'entrée au hekhal (tabernacle, arche ou armoire) placé dans le mur opposé et séparé du public par une simple grille de fer forgée. $\mathrm{Au}$-dessus du tabernacle, nous pouvons lire en hébreu le verset $20 \mathrm{du}$

\footnotetext{
${ }^{2}$ Isidore Loeb, «Les Juifs de Carpentras sous le gouvernement pontifical », Revue des études juives, tome XII, janvier-février 1886, p. 34-64.
} 
psaume 118 : « Ceci est la porte du Seigneur, les justes la franchiront ». Il contient de nombreux rouleaux de la Torah dont certains sont très anciens, en mauvais état, défectueux (psoulim) ne pouvant être utilisés lors des offices. De part et d'autre du hekhal recouvert d'un rideau moderne (parokhet), en velours de couleur rouge cramoisi avec broderies, il y a des plaques fixées au mur où sont gravés, en langue hébraïque, les dix commandements. Les boiseries recouvrant les murs et les piliers imitant le marbre, les balustres, les très nombreux lustres vieux de trois siècles et de style différents, en cristal, en argent, bronze et tôle, les rangées des bancs, constituent les éléments essentiels de ce très beau lieu de prière. Il faut rappeler un autre objet, l'une des originalités des synagogues comtadines, une petite chaise d'enfant, de style et époque Louis XVI, recouverte de velours rouge, fixée dans une niche à droite du hekhal. Elle symbolise le siège du prophète Élie, évoquant la protection de ce prophète annonciateur du Messie, lors des circoncisions. Les murs latéraux droit et gauche, qui relient le mur où est placé le hekhal et le mur où se trouve la porte d'entrée, sont ajourés par trois ouvertures demi-circulaires équipées de grilles en bois, des claustra, derrières lesquelles les femmes suivaient jadis les offices dans les couloirs (étroits), appelés ezrat nachim (emplacement de femmes).

À gauche du hekhal, sur une sellette, nous découvrons un splendide caléou ou hanoukya, une lampe à huile en bronze massif du XVII ${ }^{\mathrm{e}}$ siècle (offerte par la confrérie de l'aumône en 1618), où sont logées huit parties creuses qui reçoivent l'huile d'olive vierge avec une mèche, allumées pendant les huit soirs de la fête de Hanouka, commémorant la victoire des Maccabées et le rétablissement de la liberté religieuse et de l'indépendance politique. Le plafond simule la voûte céleste étoilée, tandis que des troncs pour diverses aumônes (dont celle de Jérusalem) se trouvent à droite et à gauche de la porte d'entrée à laquelle on accède par un escalier monumental placé à l'origine à l'extérieur du bâtiment, mais qui a dû être rentré car les autorités de tutelle l'avaient jugé trop imposant.

Sur une terrasse extérieure, derrière l'édicule du tabernacle communiquant avec la salle du culte par une porte, à gauche du mur est, on célébrait la fête de Soukot (des Cabanes), en y construisant une souka (cabane) traditionnelle.

Dans le sous-sol de la synagogue on trouve, hormis l'ancienne pièce de prières pour les femmes, la piscine rituelle et les vestiges de la fabrique des pains azymes. On peut visiter le bain rituel juif, le mikvé, en y accédant par un escalier, le bassin couvert par une belle voûte du XIV ${ }^{\mathrm{e}}$ siècle est 
alimenté par un affluent de la Sorgue. Utilisé autrefois par la fiancée à la veille des noces et pour la purification mensuelle des femmes, le mikvé n'est plus utilisé depuis très longtemps. Appelé en provençal cabussadou, dérivé du cabesso ou cabussa signifiant tête, car les femmes devaient s'y immerger la tête la première, cette piscine rituelle fut considérée par Frédéric Mistral comme la septième merveille de Carpentras...

Hormis le mikvé, il subsiste différentes pièces où l'on fabriquait les pains azymes, matzot en hébreu et coudoles en judéo-provençal, pour la fête de Pessah, la Pâque juive. Il convient de mentionner plusieurs tables de pierres, parmi lesquelles l'une qui porte le nom du donateur, et la date : Gad de Digne, 1652. C'est là qu'on pétrissait la pâte en l'aplatissant à l'aide de grands leviers de bois dont l'un est toujours en place, ainsi qu'une installation spéciale à roues dentées par où la pâte passait et était coupée en plaquettes perforées. De même, il subsiste encore les fours où l'on faisait cuire les coudoles. La fabrique du pain azyme a fonctionné jusqu'à la Première Guerre mondiale, une grande partie de la production ayant été exportée bien au-delà des frontières du Comtat.

\section{La synagogue de Cavaillon}

\section{Données historiques}

Dans la ville de Cavaillon, la plus petite des arba kehilot, la carrière fut délimitée en 1453, par le cardinal-légat Pierre de Foix, près des remparts nord-est de la ville : une petite rue unique - de la Juiverie donnant côté sud vers la rue Fabrice, aujourd'hui rue de la République, avec des maisons moins hautes qu'à Carpentras, en constituant un carré d'environ cinquante mètres de côté. La rue de la Juiverie - aujourd'hui rue Hébraïque - courte mais large impasse, s'étire jusqu'à la synagogue sous laquelle elle passe en voûte, servant de sortie à la carrière. Une deuxième porte à l'extrémité nord de l'actuelle rue Hébraïque fut construite seulement en 1774, lorsque fut terminée aussi la nouvelle synagogue dont les travaux avaient commencé deux années plus tôt. Ce fut sur l'emplacement d'un premier sanctuaire datant de 1494 que les entrepreneurs Antoine et Pierre Armelin, père et fils, furent chargés des maçonneries de la nouvelle construction, le serrurier cavaillonnais François Isoard de l'exécution des travaux de ferronnerie, et le sculpteur de L'Isle-sur-la-Sorgue, Jean Joseph Charmot, des boiseries intérieures et des sculptures en pierre et en plâtre. 


\section{Description de la synagogue}

De proportion plus réduite qu'à Carpentras, mesurant à l'intérieur $8,40 \mathrm{~m}$ de long, 7,20 $\mathrm{m}$ de large et $8,30 \mathrm{~m}$ de hauteur, la synagogue de Cavaillon monument historique (comme celle de Carpentras) depuis 1924, a été restaurée dans les années 1990, grâce au concours financier de la ville, de l'État, de la Fondation du Judaïsme français et de l'Association cultuelle israélite de Carpentras. On accède à cette synagogue de la rue Hébraïque par un escalier masqué par une maison voisine qui mène d'abord vers une terrasse munie d'une balustrade en belles ferronneries.

La porte d'entrée, de style Louis XV, qui se trouve sur la face méridionale possède de belles sculptures et, au-dessus d'elle, dans un cartouche en pierre est gravé le verset 20 du Psaume 118 que nous avons déjà retrouvé au-dessus du hekhal, à l'intérieur de la synagogue de Carpentras, et l'année hébraïque de la construction : 5534 (1774). À droite de cette porte, une fenêtre aveugle comporte six troncs à panneaux de fer en deux rangées verticales où nous pouvons lire de haut en bas, côté droit, les inscriptions hébraïques: Jérusalem, Metaharim (Purificateurs), Tibériade et, côté gauche, Hebron, Tzedaka (Aumône pour les pauvres) et Safed. Ainsi les deux caisses d'offrandes destinées à la communauté cavaillonnaise sont entourées par celles affectées aux quatre villes saintes d'Israël.

L'ordonnancement intérieur est identique à celui de la synagogue de Carpentras : le hekhal est encastré dans le mur et comporte sept niches où étaient placés les rouleaux de la Torah (aujourd'hui il n'y en a aucun) : le fauteuil du prophète Élie (qui date de 1774, don de Jehouda fils de Pinhas en 5534 d'après l'inscription hébraïque mentionnée sur le dossier) est placé à droite du hekhal, sur un socle où sont représentés des nuages; la tribune de l'officiant, ornée d'une suite de guirlande de roses et de coquilles, se retrouve en face du hekhal, du côté du mur opposé, en hauteur (on y accède par un double escalier avec rampe en fer forgé) et est soutenue par quatre colonnes en bois peint en faux marbre. C'est là que l'on peut admirer la tebah (pupitre) surmontée d'un baldaquin et un magnifique chandelier à sept branches en bois sculpté fixé dans la remarquable grille en fer forgé qui délimite la tribune du côté de la salle de prière. Rappelons enfin les sept lustres en bronze massif qui sont accrochés au plafond, ainsi que des lampes à huile, en tôle de fer peinte, suspendues par des chaînettes devant le tabernacle entouré par une belle grille en fer forgé. L'emplacement des femmes (ezrat nachim) est prévu dans la tourelle accolée au côté nord de la synagogue et qui faisait partie de l'édifice 
antérieur. Les dépendances habituelles des lieux du culte juif sont également présentes: le mikvé au sous-sol d'une cour (de l'immeuble Jouve), bordant l'escole du sud-est, et la boulangerie au rez-de-chaussée, au-dessous de la synagogue. Il s'agit d'une salle rectangulaire de 7,10 m de long, 5,20 m de large et 2,95 m de hauteur, transformée en 1963 en un musée judéo-comtadin. Lors d'une visite en 1993, j'ai pu y admirer le tabernacle (armoire) reconstitué de la synagogue primitive et trois pierres tombales dont celle d'Abraham Lisbonne, ancien président de la communauté, à droite du mur où l'on perçoit l'intérieur de l'ancien four. Dans plusieurs vitrines étaient présentés des objets utilisés dans la liturgie juive : des ornements de la Torah, les rimonim, des coupes pour le kidouch (bénédiction sur le vin), des shofarim (cornes de bélier) utilisés pour les fêtes de Yom Kippour et Roch Hachana, des hanoukyot (lampes à huiles pour la fêtes de Hanouka, des talit (châles de prières), des tefilim (phylactères, petites boîtes en cuir munies de longues lanières, contenant des parchemins avec des versets de la Torah et fixées, pendant la prière, au bras gauche et sur la tête), des mezouzot (étuis cylindriques renfermant un parchemin sur lequel sont écrits des versets bibliques et qui sont fixés au montant des portes), un seau à percussion doublé de velours pour la fête de Pourim, une cassette pour aumônes, etc. Enfin, un fouet de contrition, objet original qui ne se trouve nulle part ailleurs dans le Comtat. Au centre de la salle, dans deux vitrines horizontales, étaient réunis des manuscrits et des livres de prières, un contrat de mariage (ketoubah), des bibles, des ouvrages kabbalistiques, etc. Le visiteur pouvait enfin découvrir une carte d'Israël de 1713, une carte du Comtat Venaissin avec les « quatre saintes communautés », les arba kehilot, ainsi que la célèbre lampe d'Orgon (découverte en 1967 et attestant une présence juive dans la région dès le $1^{\text {er }}$ siècle avant notre ère).

Aujourd'hui quelques objets seulement se trouvent dans des vitrines installées au premier étage, dans la salle même de prière (la majorité restant dans des réserves, certains étant présentés lors des expositions ponctuelles à l'occasion des différentes fêtes juives). À la différence de la synagogue de Carpentras, aucun office n'est célébré, dans la synagogue de Cavaillon, considérée comme un musée.

\section{La synagogue de L’Isle-sur-la-Sorgue : données historiques}

La carrière de L'Isle-sur-la-Sorgue, la troisième des arba kahilot par son importance numérique et dont il ne subsiste aujourd'hui aucun vestige 
topographique notable, s'étendait sous la forme d'un trapèze irrégulier sur environ deux mille cinq cents mètres carrés. Elle était délimitée au sud par la rue Reboutade, à l'ouest par la rue de la Cavalerie où se dressait le Petit Portail, l'une des deux portes du ghetto, au nord par la rue longeant l'hôtel de ville et à l'est par la Grande Rue.

C'est un quartier, finalement, plus aéré que ses correspondants comtadins, au milieu duquel il y avait une place de la Juiverie, aujourd'hui la place aux bois, bien plus large que jadis. La deuxième porte, le Grand Portail, se situait du côté de la Grande Rue qui communiquait avec la Place de la Juiverie par l'actuelle rue Hébraïque.

L'escolo ou la synagogue de L'Isle, sérieusement endommagée sous la Révolution, fut démolie en 1856 et ne fut jamais reconstruite. Elle ressemblait aux autres synagogues comtadines et son entrée principale était embellie de part et d'autre par des pilastres dont les chapiteaux d'ordre dorique se trouvaient encore en 1938, d'après le témoignage du regretté Hugues-Jean de Dianoux, chez un artisan de la ville. Parmi les constructions annexes il y avait aussi une boulangerie avec le four, tandis que le mikvé public traditionnel faisait défaut au XVIII ${ }^{\mathrm{e}}$ siècle. À sa place une piscine rituelle existait dans une maison privée : en 1709 elle était la propriété de Ruven Cohen et de son fils Aron qui la donnèrent en arrentement à Aleotta de Carcassonne. Aujourd'hui, il ne subsiste, comme traces de la longue histoire des Juifs de cette localité hormis le nom place de la Juiverie, que le cimetière se trouvant sur le chemin de Caumont, au sud-ouest de la ville.

\section{La synagogue d'Avignon}

\section{Données historiques}

Avignon n'a pas abrité une communauté aussi importante que celle de Carpentras bien qu'elle ait disposé des structures d'une grande ville. Comme dans la capitale du Comtat, on y trouve une rue de la Vieille Juiverie qui rappelle l'existence d'un antique quartier juif situé sur la pente du Rocher des Dames, au nord de la ville, près des remparts de la Porte Ferruce. Très tôt, dès 1221, un nouveau quartier, près de l'église SaintPierre, fut imposé aux Juifs par l'évêque de la ville. Sa superficie fut amoindrie en 1570 et il avait alors la forme d'un quadrilatère irrégulier d'environ $100 \mathrm{~m}$ de l'est à l'ouest et de $95 \mathrm{~m}$ du nord au sud, à l'intérieur duquel était localisée une place dénommée « Planet des Juifs ». L'îlot juif dans la ville qui fut, par la présence effective des papes, la capitale de la 
chrétienté au XIV ${ }^{\mathrm{e}}$ siècle, était en 1599 , fort de 500 personnes d'après Thomas Platter.

Ils habitent, écrivait-il, la même rue, fermée par deux portes à ses extrémités. Ils sont placés sous la protection du gouvernement papal, qui leur fait payer de lourdes redevances et oblige d'assister tous les samedis, alternativement et par tiers, et cela sous les peines les plus sévères, à un sermon de pénitence qu'un jésuite est engagé de leur faire dans une église de la ville. Et cependant, a-t-on jamais vu, de mémoire d'homme, un Juif se convertir ?³

Le quartier juif possédait dans la première moitié du XVIII siècle, en plus des deux portes traditionnelles dans tout ghetto - la porte d'En-Bas ou Saint-Jean au bout de la rue Jacob et la porte d'En-Haut ou Saint-Pierre, au bout de la rue Abraham - et ce fut là une grande exception, une troisième issue dont il n'est plus question après 1750, la porte Calendre à l'est de la synagogue.

L'entassement et la surpopulation ont engendré, surtout au XVIII ${ }^{\mathrm{e}}$ siècle, des conditions d'hygiène difficiles et une insalubrité évidente. Certes, le botaniste Darluc considéra en 1780 la carrière d'Avignon comme la mieux entretenue par rapport aux trois autres ghettos comtadins. Pourtant, quelques années plus tôt, le médecin avignonnais Gastaldy, qui fréquentait quotidiennement la juiverie pour y soigner les malades, a reconnu que son enceinte était trop petite pour contenir le nombre de Juifs qui y habitaient :

À cause de cet entassement, le quartier est empesté par les mauvaises odeurs qui proviennent des immondices que les Juifs sont obligés de jeter par les fenêtres à la rue, ce qui provoque des maladies considérables, parce que les habitants respirent tous une atmosphère fétide, les rues étant fort étroites et les maisons fort élevées, presque toutes à cinq étages. ${ }^{4}$

La synagogue d'origine, remontant au XIII ${ }^{\mathrm{e}}$ siècle, a été entièrement détruite dans un incendie en 1845, mais elle nous est connue grâce à une description de 1765 ayant pour auteur le vicaire désigné par l'archevêque et inquisiteur de la ville, pour faire la visite des lieux, à la suite d'une demande d'agrandissement formulée par la communauté. Selon ce rapport,

\footnotetext{
${ }^{3}$ Felix et Thomas Platter à Montpellier. Notes de voyages de deux étudiants Bâlois, Montpellier, 1892. Cf. aussi Robert Caillet, Carpentras loué et bafoué, Lyon, Audin, 1951, p. 15-17.

${ }^{4}$ Danièle Iancu-Agou et Carol Iancu, Les Juifs du Midi. Une histoire millénaire, Avignon, éd. Barthélemy, 1995, p. 170.
} 
on accédait au vestibule de la synagogue par un escalier en forme de fer en cheval qui donnait dans la rue du côté nord. Il dresse le tableau suivant de la salle principale de prière :

[Le hekhal se trouve] dans le mur du Levant, dans une niche d'environ neuf pans de profondeur, sur huit de large et dix de haut qui se ferme par une porte de bois et une autre de fer au-devant de laquelle il y a un chapiteau [sic !] soutenu par deux colonnes de bois peint et doré et une balustrade de fer qui forme un petit parquet quarré. Du côté du Couchant règne une galerie qui occupe tout le fond de la pièce : elle est garnie d'une balustrade de fer et on y monte par deux rampes d'escalier de part et d'autre du baldaquin qui s'élève au milieu. La synagogue est éclairée par quatre fenêtres au Couchant et six au Midi, de dix pans de haut et cinq de large : son plafond est orné de peintures et de dorures. Elle mesure sept cannes deux pans du nord au sud et six cannes du levant au couchant. Sa hauteur est de cinq cannes six pans d'élévation, mesure prise au-dessus du membre bras qui sert d'école pour les femmes. Tout autour, le mur est recouvert d'une tapisserie en cuir doré d'environ douze pans de haut. Au midi, une porte de dix pans sur quatre mène au grand hazara ou terrasse, tandis que la porte principale de la synagogue qui la fait communiquer avec le vestibule s'ouvre du côté du Nord à deux battants. ${ }^{5}$

Il ressort, de cette description, que la synagogue d'Avignon avait les principales caractéristiques que l'on trouve dans les autres édifices cultuels comtadins. Elle comportait plusieurs pièces : hormis la salle de prière pour les hommes, il y avait la hazara, servant aux réunions, et l'école pour les femmes, une salle de prière spécifique qui leur était assignée dans une pièce du « membre bras », c'est-à-dire au sous-sol. Cette disposition a été constatée déjà en 1599 par Thomas Platter qui présente la salle basse comme « une vraie cave où un rabbin aveugle prêche aux femmes en mauvais hébreu car le dialecte des Juifs d'Avignon est mélangé de mots languedociens $»^{6}$. De même, par le voyageur bordelais Vande Brande, presque deux siècles plus tard :

Les femmes ne sont pas confondues pêle-mêle avec les hommes. Leur place est dans une salle souterraine semblable à un cachot, qui n'a de communication avec celle des Juifs que par un grillage de bois, qui leur procure un peu de clarté !?

Enfin, dans la principale salle de prière, le fauteuil du prophète Élie était présent dans une niche, comme à Carpentras. Le même bâtiment

\footnotetext{
${ }^{5}$ Ibid., p. 171.

${ }^{6}$ Felix et Thomas Platter à Montpellier, op. cit.

${ }^{7}$ Vande Brande, Relation d'un voyage de Languedoc Provence et Comtat d'Avignon, $1774,91 \mathrm{p}$.
} 
abritait aussi bien une jessuva (yechiva en hébreu), une salle réservée aux études religieuses approfondies, qu'une salle de mariages avec une cuisine attenante et un four à coudoles.

Lors de la reconstruction de la synagogue entreprise en 1766, certains baylons demandèrent aux autorités la permission pour les femmes de quitter leur lieu de prière au sous-sol pour une salle supérieure, séparée néanmoins par une grille de l'école des hommes. L'autorisation fut bien octroyée par l'inquisiteur le 26 décembre 1766, mais le projet ne se réalisa point en raison de l'opposition des traditionalistes dirigés par Salomon Delpuget, Michaël Haïn de Saint Paul et Menassé de Béziers qui n'acceptèrent aucunement la proposition de «mettre les femmes au haut de leur école et de laisser la liberté à celles qui voudraient aller en bas, ce qui serait totalement contraire à l'usage qui se pratique parmi les Juifs et qui s'est pratiqué dans tous les temps tant dans cette carrière que dans la carrière des Juifs du Comtat», d'après un acte enregistré par-devant notaire le $1^{\text {er }}$ mai $1767^{8}$. Dans la défense de leur position concernant la séparation absolue des sexes, les traditionalistes avancèrent le souci de la ferveur des prières, arguant qu'une présence féminine, même discrète, risquait de perturber les cérémonies du culte... L'aménagement de la synagogue en deux niveaux fut conservé jusqu'à la Révolution, lorsque le nombre d'habitants de la carrière s'éleva à 350 .

De l'ancienne carrière il ne subsiste que le site où se trouve la synagogue, qui comprend la place de Jérusalem (l'ancien «Planet des Juifs », dénommé place Victor Basch après 1945) et les rues Abraham et Jacob.

\section{Description de la synagogue}

La synagogue reconstruite entre 1765 et 1767, sur les plans de l'architecte avignonnais Jean-Baptiste Franque, fut malheureusement détruite en 1845, dans un incendie qui avait pris naissance au rez-dechaussée et l'ampleur des dégâts n'ayant pas permis une restauration, une toute nouvelle synagogue fut construite à la place. Financée par la ville, elle fut au départ l'œuvre de Jules Duchesne, ingénieur des Bâtiments civils, poursuivie par l'architecte de la ville Joseph-Auguste Joffroy, sur l'emplacement de l'ancien lieu du culte. Elle est entièrement différente de la précédente, bâtie sur un modèle néoclassique à la mode au XIX ${ }^{\mathrm{e}}$ siècle, inspirée par le principal temple israélite de Vienne, le Stadttempel. Son originalité, enfin, réside dans sa forme circulaire, unique en France, une

\footnotetext{
${ }^{8}$ Danièle Iancu-Agou et Carol Iancu, op. cit., p. 171.
} 
rotonde dans un bâtiment trapézoïdal, couverte d'une coupole dont le centre assure l'éclairage, l'immeuble ne possédant aucune fenêtre. D'une hauteur de $17 \mathrm{~m}$ et d'un diamètre de $12 \mathrm{~m}$, l'intérieur possède des colonnades superposées, la place des femmes se trouvant à l'étage. Au rezde-chaussée, le hekhal, en noyer verni, se trouve dans une travée de la colonnade inférieure, côté est, tandis qu'une chaire en menuiserie, suspendue à l'une des colonnes, remplace la tribune du rabbin. Devant le hekhal, est placée une tebah tout à fait particulière: deux grands chandeliers entourent, à droite et à gauche, le pupitre incliné, tandis que l'estrade pourvue de deux portes et d'une clôture semi-circulaire, contient dix places séparées par des accotoirs. C'est vers des vestibules distincts que s'ouvraient les deux portails de la façade, côté sud pour les hommes qui se dirigeaient vers la salle de prière au rez-de-chaussée, et côté nord pour les femmes qui s'orientaient vers l'étage. Actuellement il y a une seule entrée commune, une porte qui se trouve dans le mur sud. La salle de prière circulaire, qui ne possède pas le fauteuil du prophète Élie, ni d'ailleurs aucun élément semblable aux synagogues comtadines, contient quatorze colonnes d'ordre ionique, la colonnade soutenant la tribune est scandée elle aussi par quatorze colonnes d'ordre corinthien, soutenant la coupole.

\section{Quelques aspects de la vie culturelle et religieuse}

Malgré leur nombre restreint, les Juifs du pape, dont la plupart portent les noms des villes du Comtat et du Midi de la France, ont développé une vie religieuse et culturelle riche, originale par la langue, la création littéraire et la liturgie. En effet, ils avaient leur propre parler intime : le judéo-provençal ou l'hébraïco-comtadin, appelé chouadit (de l'hébreu yehudit), un mélange de provençal vernaculaire (pour la majeure partie du vocabulaire), d'hébreu et d'autres termes particuliers. L'une des dernières personnes connaissant ce parler fut Blanche Mossé, la gardienne de la synagogue de Carpentras encore après la Seconde Guerre mondiale ; aujourd'hui il a complètement disparu. Si le chouadit fut la langue orale des Juifs du pape, c'est d'abord en provençal qu'ils écrivirent leurs œuvres littéraires. Citons ici le rabbin Mardochée Astruc de L'Isle-sur-la-Sorgue qui composa La Reine Esther au XVII ${ }^{\mathrm{e}}$ siècle, une tragédie reprise et augmentée au XVIII ${ }^{\mathrm{e}}$ siècle par Jacob de Lunel, rabbin de Carpentras, et imprimée dans cette ville en 1774. L'écrivain Armand Lunel y a puisé certains éléments pour son opéra-bouffe Esther de Carpentras, avec la 
musique de Darius Milhaud. C'est en langue provençale que le célèbre "Chant du chevreau » Cansoun dou cabri ou Had Gadya du rituel de la Pâque juive est chanté à la fin de la Hagadah (récit de la Pâque) ${ }^{9}$, les deux premiers soirs de cette fête. Une production littéraire tout à fait originale est constituée par un ensemble de poésies appelées en judéo-provençal lis obros (œuvres) et en hébreu pioutim (cantiques) qui avaient la particularité de faire alterner un vers en langue hébraïque et un vers en langue provençale, constituant des pièces «farcies». Elles ont été composées pour diverses circonstances de la vie, surtout pour la circoncision et le mariage, ou pour certaines fêtes, notamment celle de Pourim et furent mises rapidement en musique.

C'est en hébreu que furent rédigés les escamot, les pinkassim, les correspondances et des œuvres littéraires, philosophiques et exégétiques. Parmi les poètes les plus réputés du XVIII ${ }^{\mathrm{e}}$ siècle, rappelons Joseph ben Abraham, Saul ben Joseph de Monteux, Mordekhaï ben Jacob de Carpentras, Gad ben Juda de Bédarride et Joseph de Meyrargues, auteur d'une ode hébraïque au pape Clément XIV. D'autres érudits se sont fait connaître dans le domaine de la philosophie (Moïse ben Mikhael de Milhaud), de l'exégèse (Josua du Caylar, Joseph de Milhaud, Moïse de Michaud et les frères Mardochée Crémieux et Salomon Hayim Crémieux), de la traduction (Gabriel de Milhaud) ou de la jurisprudence (Izaac ben Emmanuel de Lattes et David Milhaud).

Proches du monde séfarade, les Juifs du pape ont leurs propres liturgies avec des spécificités locales; il s'agit d'ouvrages restés très longtemps (jusqu'au milieu du XVIII ${ }^{\mathrm{e}}$ siècle) sous forme de manuscrits copiés par les rabbins. Le développement de l'imprimerie, dans la ville d'Avignon, permit la parution dans cette localité, en 1767, du Seder Hatamid, un rituel de prières à l'usage des communautés de Carpentras, Avignon, L'Isle-sur-la-Sorgue et Cavaillon, publié par Élie Crémieux à la demande de Mardochée Crémieux. C'est ainsi que devait naître le rite comtadin qui se maintiendra dans la diaspora des Juifs du pape, surtout dans les provinces voisines, Provence et Languedoc, particulièrement dans les villes suivantes : Aix-en-Provence, Arles, Marseille, Alès, Lunel, Montpellier, Nîmes, Pont Saint-Esprit...

Parmi les caractéristiques des rituels comtadins, il faut insister sur la présence des pioutim (cantiques), des tehinot (complaintes), des nichmatim (élégies) et des tefilot (prières) spécifiques dont les auteurs - pas toujours

\footnotetext{
${ }^{9}$ Carol Iancu, «Pessah 5780 : pour la liberté, vers l'amitié et l'entente », L'Écho des Carrières. Bulletin de l'Association des Juifs du Pape, $\mathrm{n}^{\circ} 95,1^{\mathrm{er}}$ semestre 2020, p. 10-11.
} 
connus - se recrutèrent parmi les habitants des carrières. Après la fin du régime pontifical, les carrières se vidèrent et l'intégration des Juifs du pape à la société française s'effectua rapidement. Aux $\mathrm{XIX}^{\mathrm{e}}$ et $\mathrm{XX}^{\mathrm{e}}$ siècles, beaucoup de Juifs d'origine comtadine ont joué un rôle important en France : Jassuda Bédarride, maire d'Aix-en-Provence en 1848, AdolpheIsaac Crémieux, ministre de la justice en 1848 et auteur du décret émancipant les Juifs d'Algérie, Alfred Naquet, député à l'Assemblée Nationale qui fit voter la loi sur le divorce (1884), Bernard Lazare, écrivain et journaliste, le premier à démontrer l'innocence du capitaine Dreyfus, Mardochée Georges Valabrègue, général d'armée, René Cassin, prix Nobel de la Paix (1968), l'écrivain Armand Lunel, le musicien Darius Milhaud, etc.

\section{Orientation bibliographique}

Bonan Aurélie, Patrimoine juif d'Avignon et du Comtat. Parcours du

Patrimoine, 425, Lyon, Lieux Dits, 2019.

Caillet Robert, Carpentras loué et bafoué, Lyon, Audin, 1951.

Dumoulin André, Un joyau de l'art judä̈que français. La Synagogue de Cavaillon, Klinsieck, 1970.

Dianoux Hugues-Jean de, Les communautés juives du Comtat et de l'État d'Avignon d'après leurs statuts (1490-1790), thèse, École des Chartes, 1938.

Iancu Carol (direction), Armand Lunel et les Juifs du Midi, Montpellier, Université Paul Valéry, 1982, 350 p.

Iancu Carol, Itinéraire juif du Midi, film documentaire, Toulouse, France 3 Sud, 1998.

Iancu Carol et Danièle, Route du patrimoine juif du Midi de la France, Avignon, Comité Départemental du Tourisme de Vaucluse, 1992.

Iancu Danièle et Carol, Les Juifs du Midi. Une histoire millénaire, Avignon, éd., Barthélémy, 1995, 351 p. 
Iancu Carol, «Pessah 5780 : pour la liberté, vers l'amitié et l'entente », L'Écho des Carrières. Bulletin de l'Association des Juifs du Pape, $\mathrm{n}^{\circ} 95,1^{\mathrm{er}}$ semestre 2020, p. 5-11 (en annexe : le «Cansoun dou cabri » " Chant du chevreau », en provençal et en traduction française).

Milesi Gérard, «Les Juifs de L'Isle-sur-la-Sorgue au XIX ${ }^{\mathrm{e}}$ siècle», Mémoire de maîtrise sous la direction de Carol Iancu, Université Paul Valéry, 2001.

Moulinas René, Les Juifs du pape en France. Les communautés d'Avignon et du Comtat Venaissin aux XVII et XVIII siècles, Toulouse, Privat, 1981.

Mrejen-O'Hana Simone, «Les pinkassim de Carpentras au regard du Saint-Siège. Le Sefer ha-Yahas (1736-1769) d'Élie Crémieux ", Bulletin du Centre Français de Jérusalem, 2005, n 16 , p. 45-75.

Nahon Gérard, "Ode hébraïque de Joseph de Meyrargues au pape Clément XIV, Carpentras, c.a. 1774 », Sefarad, t. LII, fasc. 2, 1992, p. 513-526.

Roth Cecil, "The liturgies of Avignon and the Comtat Venaissin », Journal of Jewish Bibliography, 1, 1939, p. 99-105.

Schwarzfuchs Simon, "Échos hébraïques du Comtat et de ses carrières », dans Carol Iancu (direction), Armand Lunel et les Juifs du Midi, op. cit., p. 207-213.

Szajkowski Zosa, «HaYehudim beArba haKehilot shel haProvintzia haAfifiorit beDrom Tzarfat. Hoveret uMismakhim mudpasim meHaMea haCheva-esre ad thilat haMea haTicha-esre », (Les Juifs des quatre communautés de la Provence pontificale en France du sud. Brochure et documents imprimés du dix-huitième siècle jusqu'au début du dix-neuvième), Kiriat Sepher, 32, 1956-1957, p. 205-210 et p. 349-356. 


\section{Synagogue de Carpentras}

(photos de Carol Iancu)

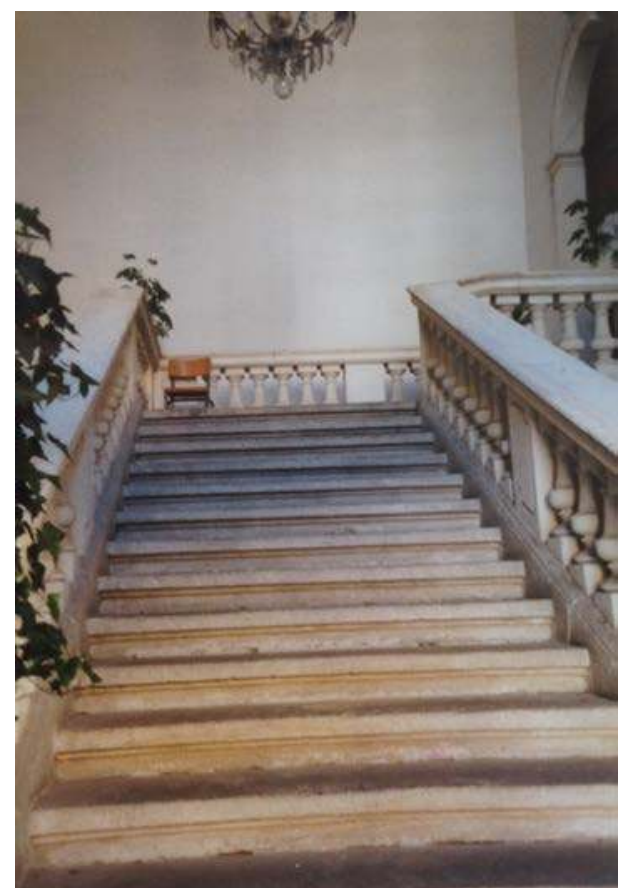

Escalier intérieur vers la salle de prière

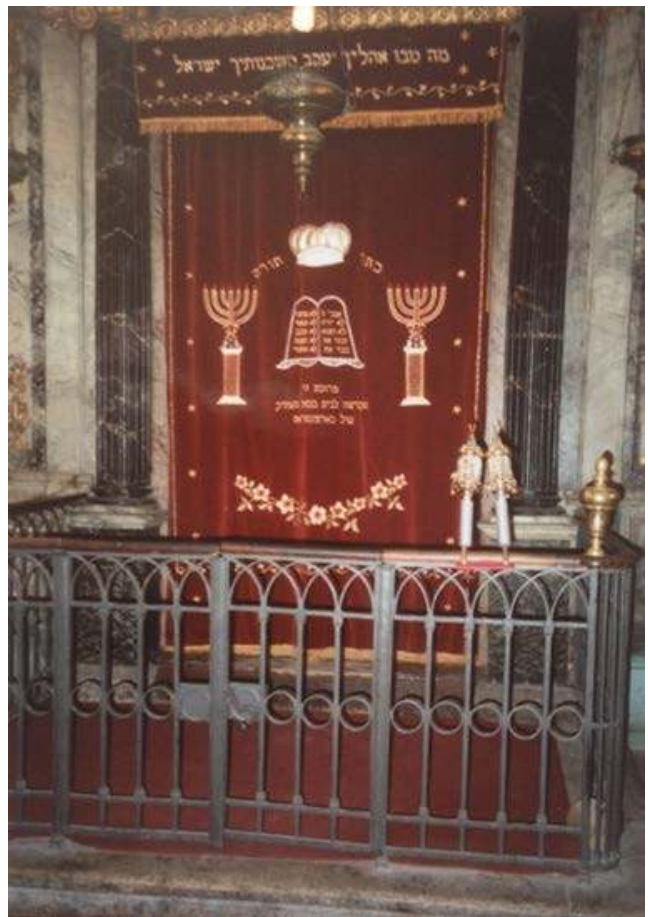

Hekhal couvert d'un rideau (parokhet) moderne 


\section{Synagogue de Carpentras}

(photos de Carol Iancu)

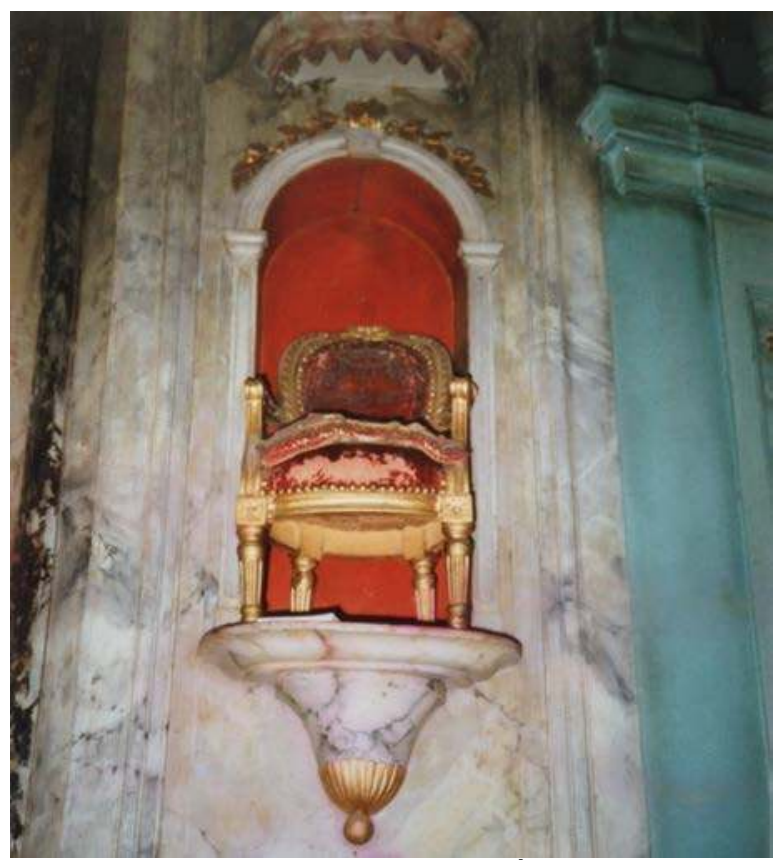

Fauteuil du prophète Élie

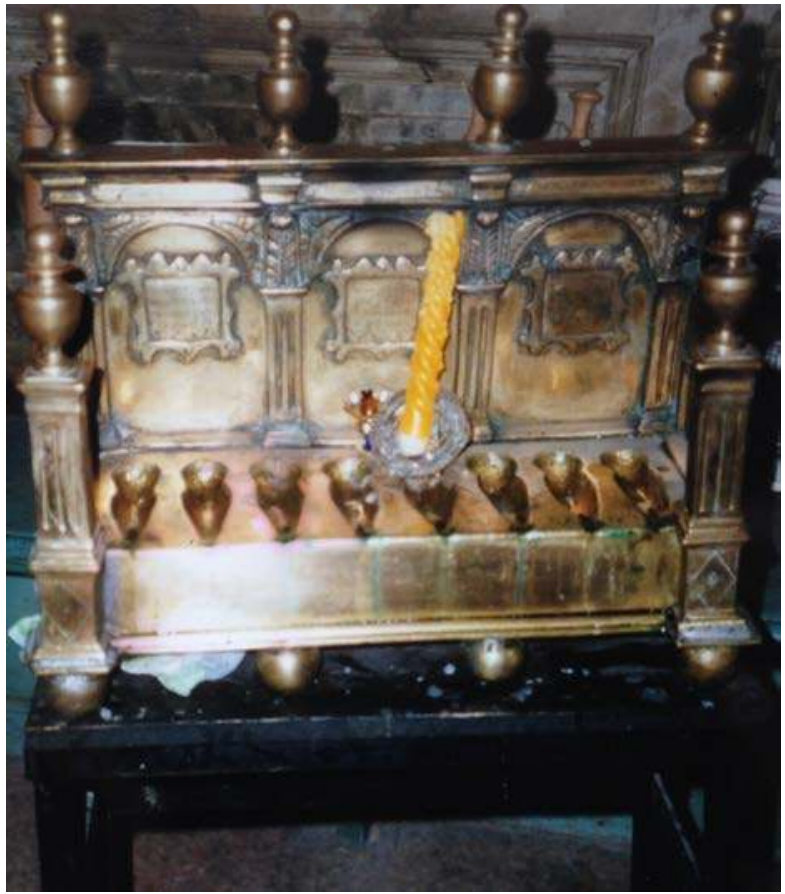

Caléou (lampe de Hanouka) (1618) 


\section{Synagogue de Cavaillon}

(photos de Carol Iancu)

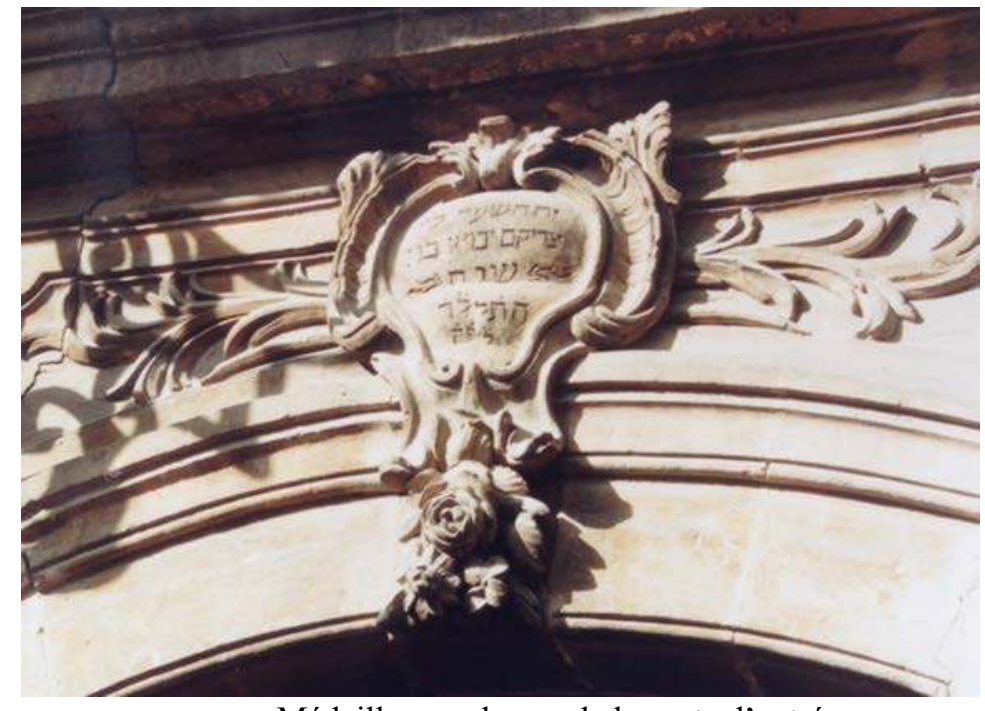

Médaillon au-dessus de la porte d'entrée

En hébreu : le psaume 118,20 et l'année hébraïque 5534 (1774)

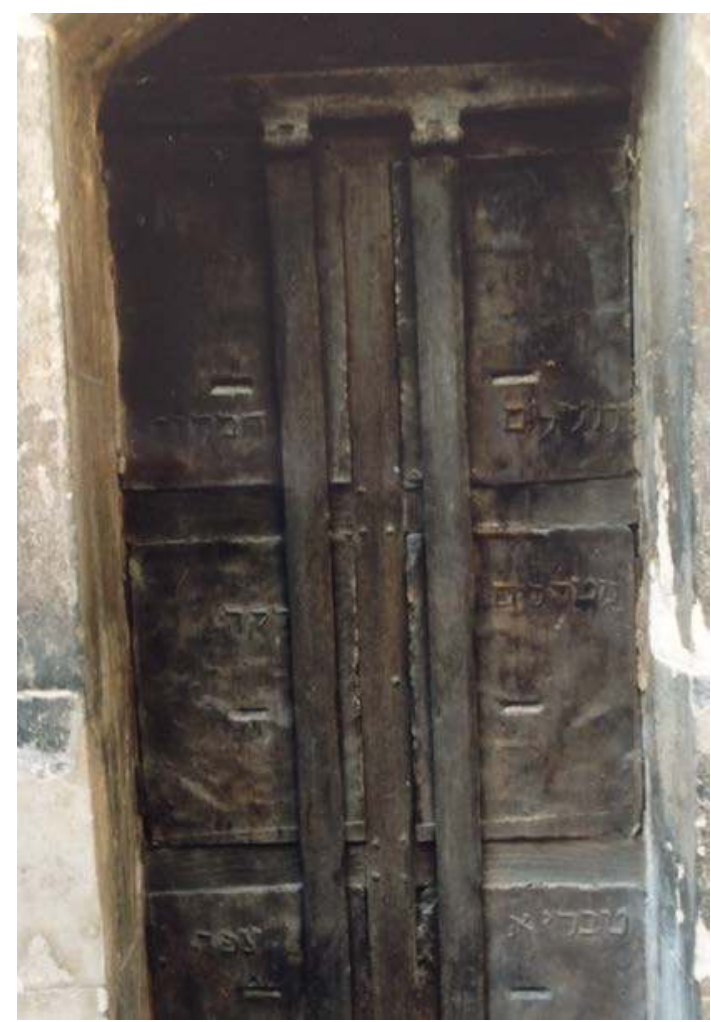

Fenêtre avec troncs pour aumônes 


\section{Synagogue de Cavaillon}

(photos de Carol Iancu)

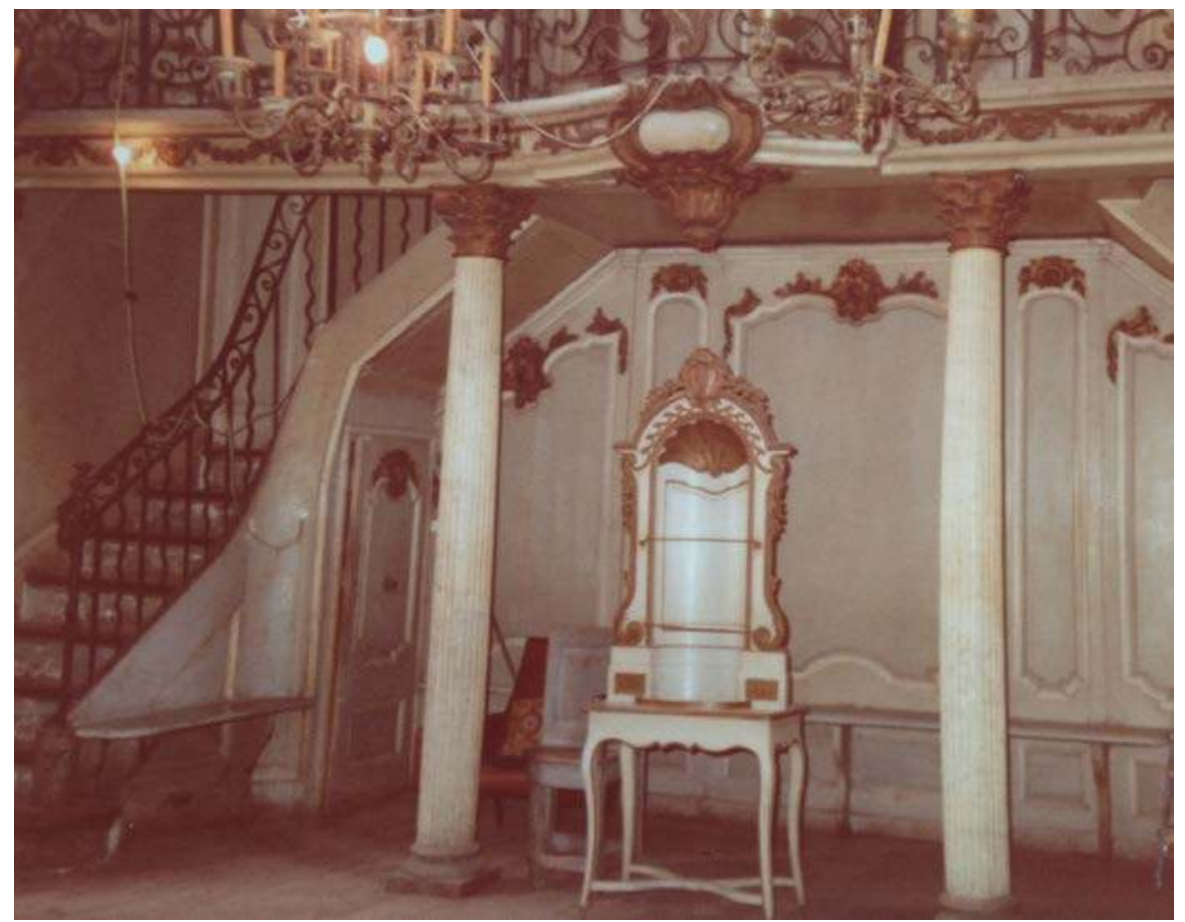

Escalier vers la tebah et table avec niche ornée pour la Tora

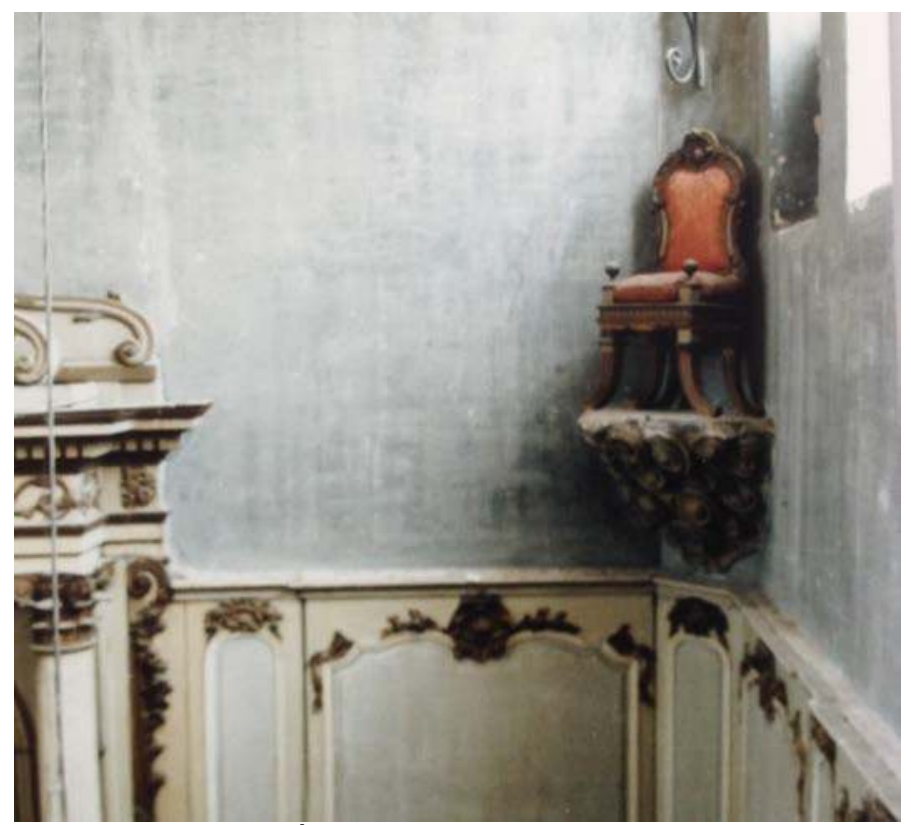

Siège du prophète Élie, avant la restauration de la synagogue 


\section{Synagogue d'Avignon}

(photos de Carol Iancu)

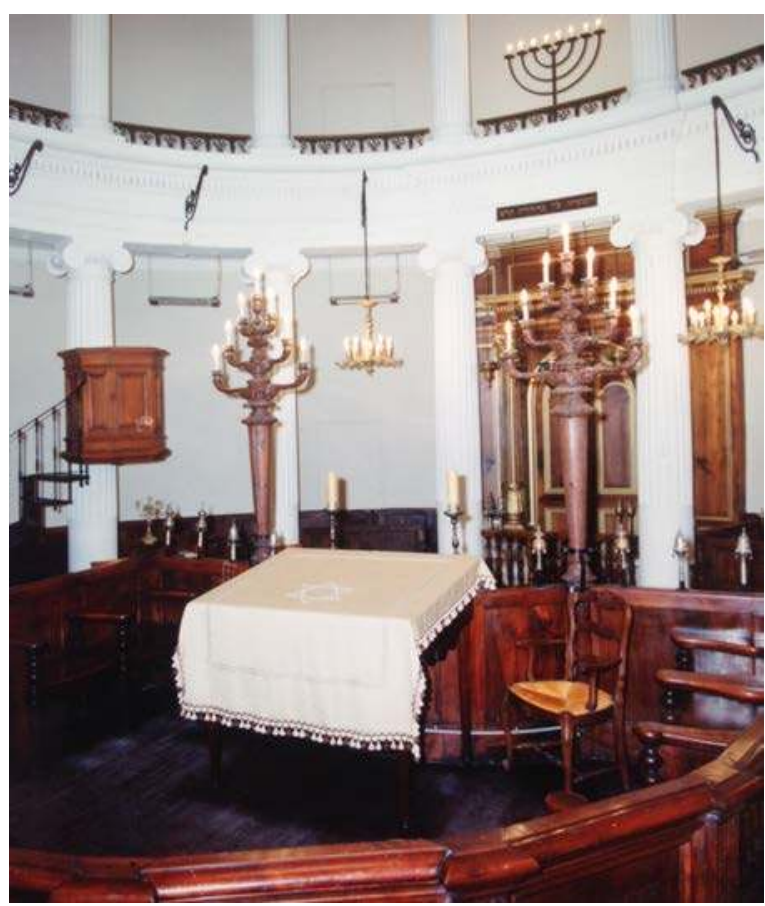

Tebah, chaire, chandeliers et hekhal

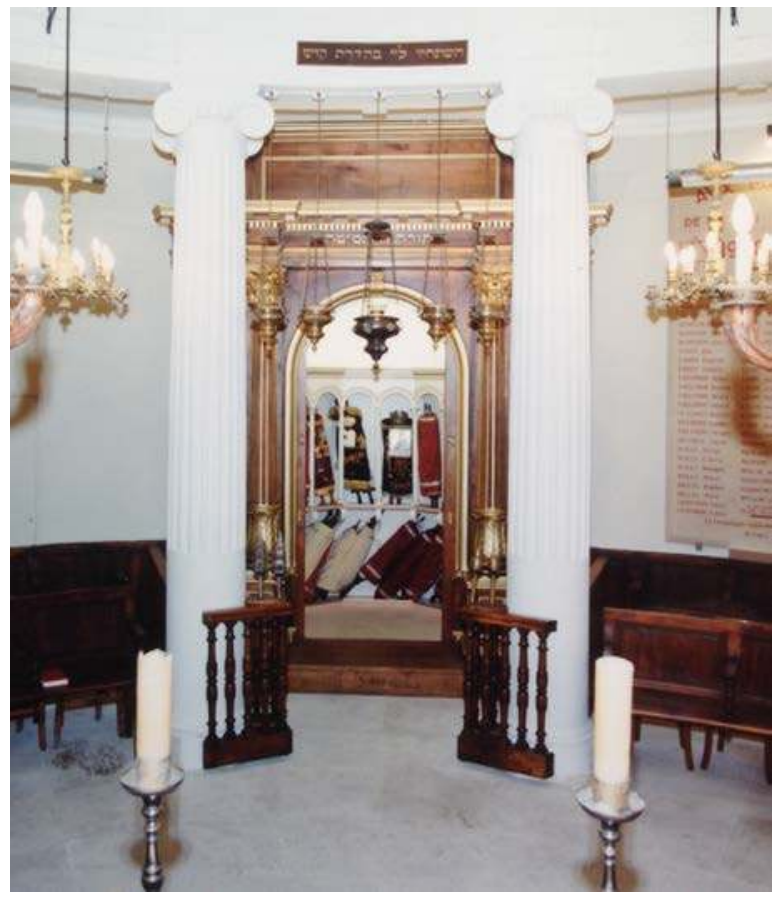

Intérieur du hekhal 